\title{
INITIATION FROM A POINT ANODE IN A DIELECTRIC LIQUID
}

\author{
By: \\ M. Kim \\ R.E. Hebner
}

IEEE Transactions on Dielectrics and Electrical Insulation (in review)

PR 430

Center for Electromechanics

The University of Texas at Austin

PRC, Mail Code R7000

Austin, TX 78712

(512) $471-4496$ 


\title{
Initiation from a Point Anode in a Dielectric Liquid
}

\author{
M. Kim and R. E. Hebner \\ University of Texas at Austin \\ Austin, TX 78712, USA
}

\begin{abstract}
Many previous studies of electric breakdowns in dielectric liquids in point-plane geometry have examined the relationships among the breakdown structure (or speed), the electrode geometry (point radius, gap length), and/or voltage. This paper explores the hypothesis that, for streamers initiating from a point anode, the critical volume model used for similar geometry in gaseous dielectrics is useful in liquids. The assumption of the critical volume is shown to be consistent with experimental data. Specifically, a critical volume of $0.4-1.0 \mu \mathrm{m}^{3}$ is consistent with the location of streamer initiation, with the independence of the initiation voltage for the $2^{\text {nd }}$ anode mode from the tip radius for sharp tips, and the measured free paths of electrons in cyclohexane for the energies of interest.
\end{abstract}

Index Terms - Dielectric liquids, cyclohexane, breakdown, mean free path

\section{INTRODUCTION}

PREVIOUS studies [1-4] of electric breakdown in dielectric liquids in positive-point point-plane geometry have shown that the initiation voltage for a prebreakdown streamer is a function of both tip radius and gap spacing. The initiation of electrical breakdown under these conditions in an insulating liquid is expected to be an electron-dominated process. Fundamentally, the force, F, on an electron of charge, e, is given by

$F=e E$,

where $E$ is the electric field. Thus, the energy deposited in the fluid is proportional to the kinetic energy the electron accumulates before colliding with a molecule, i.e., $e E \cdot \lambda$, where $\lambda$ is the path length over which the electron was accelerated. If the energy transferred in the collision between an electron and a molecule is less than the ionization energy for the molecule, the collision energy can be absorbed as heat, ultimately leading to localized boiling. For energy transfers greater than the ionization energy, a streamer can be initiated. As noted previously [5], the lower energy picture is consistent with the formation of the $1^{\text {st }}$ anode mode, while the $2^{\text {nd }}$ anode mode is consistent with more energetic collisions.

The experimental work of Yamashita, Yamazawa, and Wang [3] and that of Gournay and Lesaint [4] provide good data to help clarify the role of electron energy on streamer initiation. Each of these studies was conducted in cyclohexane, each used a point-plane electrode system (albeit with some differences in the geometry), and both obtained consistent results. Of particular interest in this work is the data showing that for very sharp tips either a $1^{\text {st }}$ anode mode or a $2^{\text {nd }}$ anode mode could be the initiating streamer. Moreover, when the process was initiated by a $1^{\text {st }}$ anode mode, the initiation voltage increased with increasing tip diameter, a trend consistent with the change in voltage required to attain the same maximum electric field. If the process were initiated by a $2^{\text {nd }}$ anode streamer, however, the initiation voltage was independent of the tip radius for small tip radii. This means that the maximum field was quite different while the streamer initiation voltage was the same.

The present work shows these results are consistent with an electron-energy model of the process if one invokes a critical volume model analogous to the one that has found applicability in the understanding of the processes in gaseous breakdown. A confounding factor is that, in liquids, high Rydberg states have a radius such that electrons in these states interact with neighboring molecules. This does not occur in gases at room temperature unless the pressure exceeds about $10^{6} \mathrm{~Pa}$ [6]. Thus the electron-molecular interaction is simpler in these gases.

\section{CRITICAL VOLUME}

In gaseous breakdown, it has been shown that, for given geometry and voltage, the probability $d P$ that a first critical avalanche will be created during time interval $d t[6]$ is given by 


$$
d P=(1-P) \int_{\Gamma_{c}} \frac{d n_{e}}{d t} P_{0} d \Gamma d t
$$

where $d n_{e} / d t$ is the electron production rate, $P_{0}$ is the probability that electrons will remain active, and $\Gamma$ is the volume. The critical volume $\Gamma_{c}$ in gases is defined by two contour lines of constant electric field. The line furthest from the point electrode is the line at which an electron at that surface experiences a high enough electric field to initiate ionization upon collision with an atom or molecule. The contour nearer the electrode is the surface of maximum field strength at which an electron does not have adequate distance to produce an avalanche, because too few collisions are possible before impacting the anode.

The solution of (2) is given by

$$
P(t)=1-\exp \left(\int_{t_{0}}^{t_{s}} \int_{\Gamma_{c}} \frac{d n_{e}}{d t} P_{0} d \Gamma d t\right),
$$

where $t_{s}$ is the statistical time lag and $t_{0}$ is the time at which the streamer inception level is exceeded. The fundamental quantity required for calculating the statistical time lag is the rate of production of initiatory electrons. The production rate of effective electrons is given by

$$
R_{e}(t)=\int_{\Gamma_{c}(t)} \frac{d n_{e}}{d t} P_{0} d \Gamma \text {. }
$$

Key factors for streamer initiation are growth of $\Gamma_{c}$ with applied voltage and $d n_{e} / d t$.

A different assumption has also been used in dielectric liquids with a high degree of success. Kupershtokh and colleagues $[7,8]$ have postulated that, while the streamer is propagating, the field in the vicinity of a streamer tip should be expressed as $E_{0}+\delta$, where $E_{0}$ is the electric field strength necessary for breakdown and $\delta$ is a fluctuation in the electric field caused by changes in the local dielectric constant due to such factors as density fluctuations or molecular excitations. Using this assumption, they are able to develop a stochastic model that produces streamer growth patterns that are consistent with the growth patterns that have been observed in experiments.

There is no discrepancy between the field fluctuation assumption and the electro-optical observation that the field between the streamer and the electrode to which it is propagating can be modeled assuming the streamer is a conductor [9]. The experimental observations did not have sufficient precision to detect small, highly localized fluctuations in the electric field.

There is a discrepancy, however, between the field fluctuation model and the critical volume model that embeds the stochastic behavior in the production of electrons rather than in electric field variations [10]. While it is likely that the higher density of a liquid than a gas leads to variations in the local dielectric constant, there is not sufficient evidence to determine which stochastic process dominates. As shown in
(3), the production of the necessary electrons to initiate a streamer is controlled by both the rate of free electron production and the electric field. They are very difficult variables to separate experimentally.

This work demonstrates that the critical volume model provides a framework in which different experimental results can be reconciled. It does not, however, show which fundamental process dominates under the conditions tested. That is still an unresolved question.

\section{RESULTS}

In both [3] and [4], the variations of initiation voltage with changes in point diameter were observed for the $1^{\text {st }}$ anode mode streamers, but a constant initiation voltage was seen if the initiation was via the $2^{\text {nd }}$ anode mode. To obtain greater insight into the underlying processes, the electric field distributions with the geometries from $[3,4]$ were calculated in cylindrical coordinates assuming azimuthal symmetry. The detailed tip configurations used in the two experimental studies are shown in Figure 1. The critical volume with equifield surfaces as boundaries was also calculated.

\subsection{Case 1}

The calculated electric field strengths for the geometries in Figure 1 were compared with the data from [3] in Figure 2. To verify the result, the computation was repeated using a boundary element approach. Figure 2 shows the two computational approaches yield very similar results.
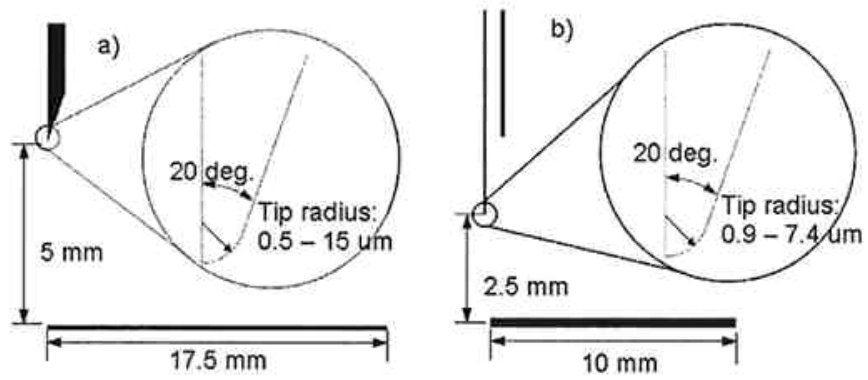

Figure 1. Analysis configurations of a) [3] and b) [4]. a)

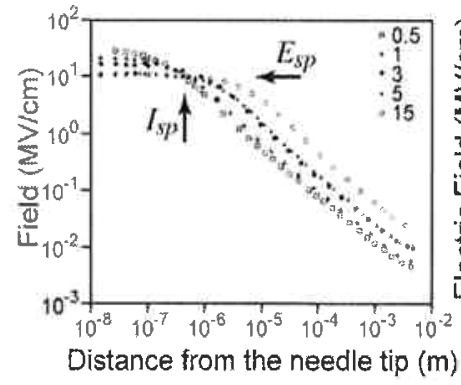

b)

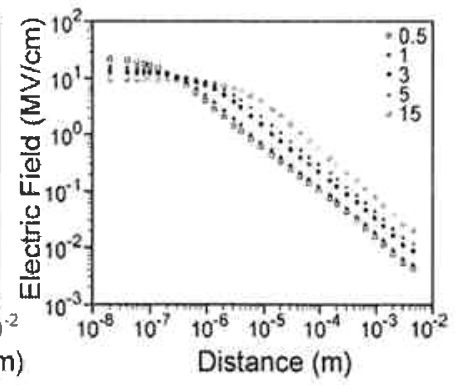

Figure 2. Electric field distribution from a) [3] and b) calculated for this work. The consistency suggests that both computational approaches are valid. 
An important observation made in [3] is that, for the geometry used, the calculated electric field distribution along the axis of symmetry shows a point of nearly constant electric field strength for all tip radii studied. This point is a few tenths of a micrometer from the tip. The initial work was done using a finite element approach [10] for the calculation. This nearly constant region, however, is shown on a log-log plot. Therefore, a more precise analysis of the field distribution near the tip is needed to draw more reliable conclusions.

First of all, let us define a criterion of the electric field, $\mathrm{E}_{\max }$, which is the maximum electric field of the $0.5 \mu \mathrm{m}$ tip radius case at a voltage of $9.3 \mathrm{kV}$.

For the geometry of Figure 1a), Figure 3 shows, for various tip radii, the distance from the tip that the field strength is at specified percentages of $\mathrm{E}_{\max }$. The sign of the slope for the electric field strength changes with increasing tip radius changes between $40 \%$ and $50 \%$ of $\mathrm{E}_{\max }$. Consequently, the smallest variation of the electric field strength with tip radius occurs between $40 \%$ and $50 \%$ of $\mathrm{E}_{\max }$. The distance from the tip is between 0.2 and $0.4 \mu \mathrm{m}$, a value consistent with the calculations summarized in Figure 2. This suggests that there might be a consistent field for initiation having a size in which the initiation can be detected optically.

A confounding factor, however, occurs due to the finite rise time of an applied voltage pulse. Figure 4 shows four presumed critical volumes, i.e., the inner bounds of the surfaces of constant electric field. If the outermost line is assumed to coincide with the critical volume at the peak voltage for a given geometry, the inner three lines are boundaries of critical volumes that would be produced (at the same minimum field strength) during the rise or fall of the applied voltage pulse.

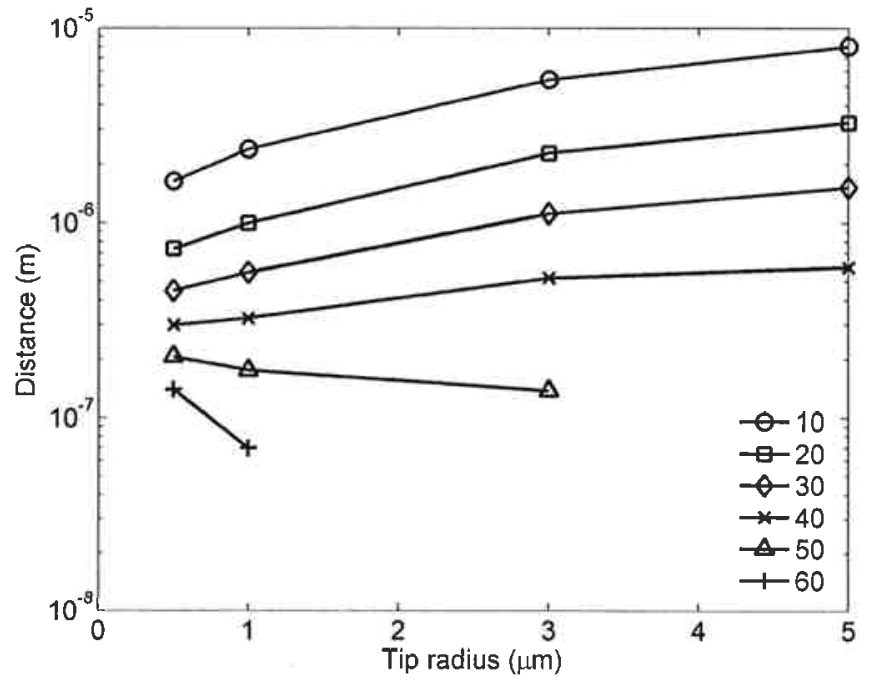

Figure 3. The calculated electric field strength as a function of tip radius for various percentages of $E_{\max }$ with the geometry of Figure 1a). This calculation shows that for the conditions of the study, the slope of field strength changes sign between $40 \%$ and $50 \%$ of $\mathrm{E}_{\max }$.

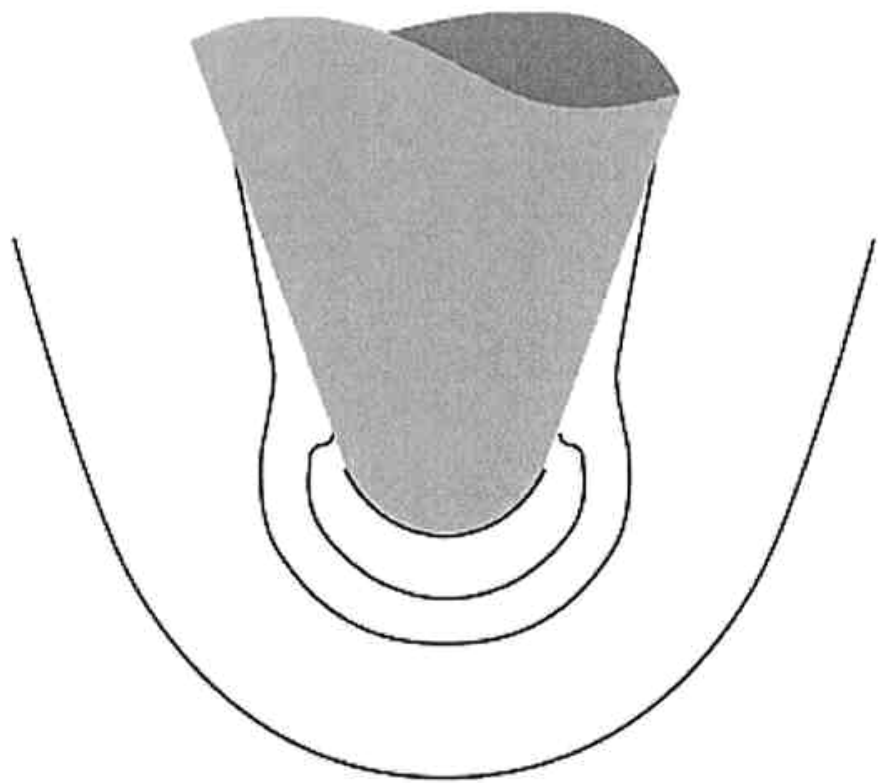

Figure 4. Contours of constant electric field strength for a point anode near the tip. While the maximum field strength occurs at the tip, the critical volume can encompass more of the tip.

One immediate conclusion from this is that if the voltage were raised on time scales comparable to the production rate of electrons (or field fluctuation times), initiation of a streamer could occur at the tip, on the side of the shaft, or both. Figure 5 shows streamers, in several liquids including cyclohexane, not only at the electrode tips, but also at the side of the electrode for both positive and negative streamers. The upper eight frames show negative streamers and the bottom ones, positive streamers. The streamers from the side are shown in both cases. The tip radius for this data is $50 \mu \mathrm{m}$. This experimental data is consistent with a critical volume for initiation rather than initiation at maximum field strength.

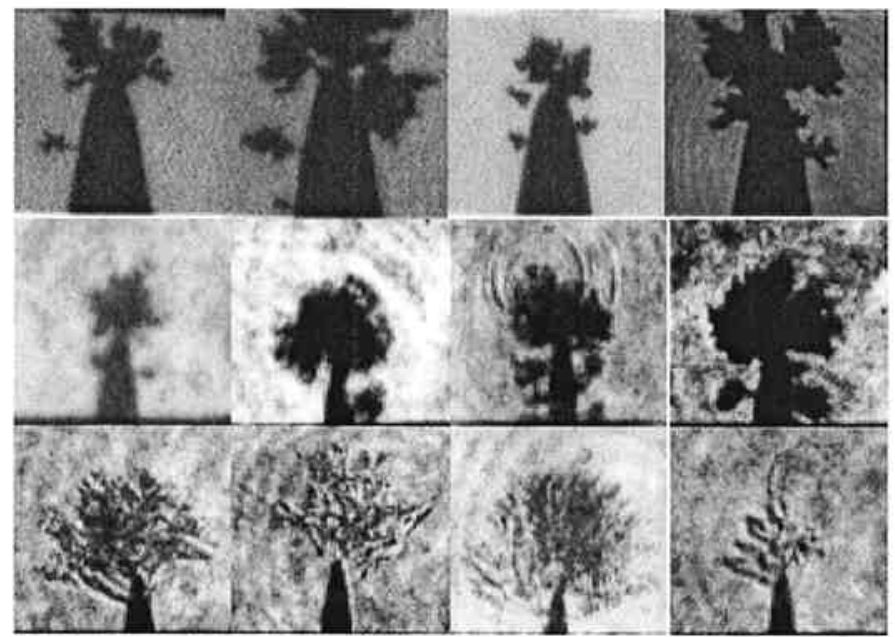

Figure 5. Streamer initiation. The negative streamers in cyclohexane in the top row, negative streamers in $\mathrm{DC} 10$, hexane, $\mathrm{n}$-hexane, and toluene in the second row, and positive streamers in n-hexane in the bottom row show that, as would be predicted from Figure 4, streamers are not limited to the electrode tip. Since these were taken with a rising voltage waveform, the initiating volume could be larger than the minimum critical volume. 
The variation of the size of the critical volume with changes in tip radius was calculated for a range of electric field strengths and the results are shown in Figure 6. The critical volume increases if the electric field defining it is between $10 \%$ and $40 \%$ of $\mathrm{E}_{\max }$, and decreases steeply if the field is greater than $50 \%$ of $\mathrm{E}_{\max }$. For an electric field of approximately $50 \%$ of $\mathrm{E}_{\max }$, the critical volume is between 0.4 and $1.0 \mu \mathrm{m}^{3}$.

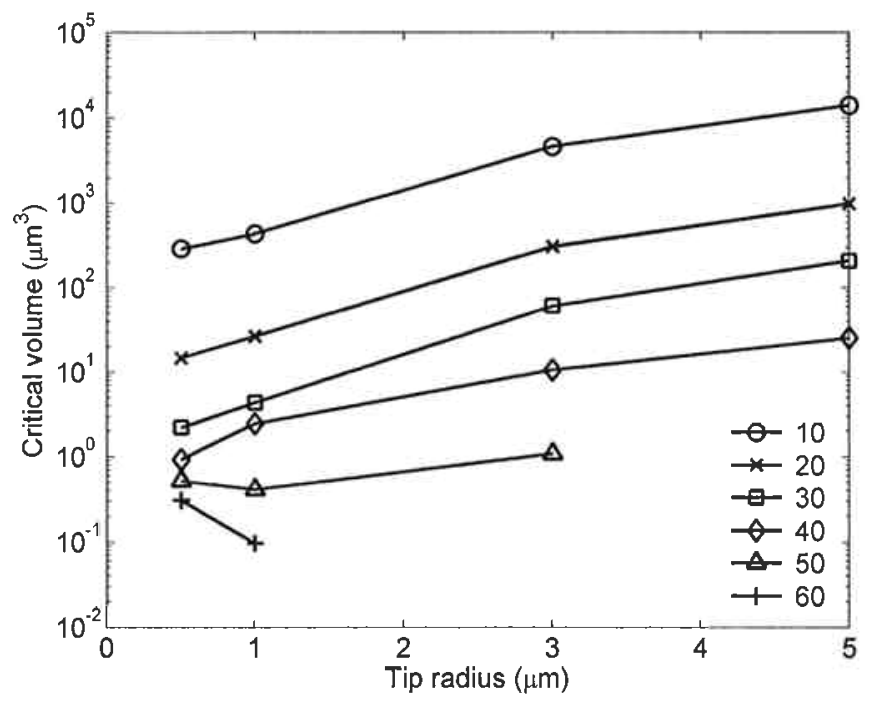

Figure 6. Critical volume with chosen electric fields in Case 1.

\subsection{Case 2}

The preceding analysis was done for the electrode geometry used by Yamashita, et al [3]. To confirm that the conclusions are not limited to that single experiment, the analysis was also performed for the experiments of P. Gourney and O. Lesaint [4]. In this analysis, the boundary element computation was able to account for both the finite size of the plane electrode and for the steel tube used as a guard on the point electrode. Figure 7 , which compares the originally published data with the results of this analysis, shows the analysis is well matched to the measured data. Gourney and Lesaint presented the measured breakdown voltage at two different sizes of the plane electrode and showed that as the plane electrode became larger, the measured data agreed with an analytical solution for an infinite plane.

For the configuration of Figure 1b), Figure 8 shows the distance from the tip that a given percentage of $E_{\max }$ occurs for various tip radii. The sign of slope for the electric field strength changes between $30 \%$ and $40 \%$ of $\mathrm{E}_{\max }$. Unlike Case 1 , the smallest variation of the electric field strength with tip radius occurs between $30 \%$ and $40 \%$ of $\mathrm{E}_{\max }$. The distance from the tip, however, is between 0.2 and $0.4 \mu \mathrm{m}$, a value consistent with the calculations summarized in Figure 2, and with the results of Case 1 in Figure 3.

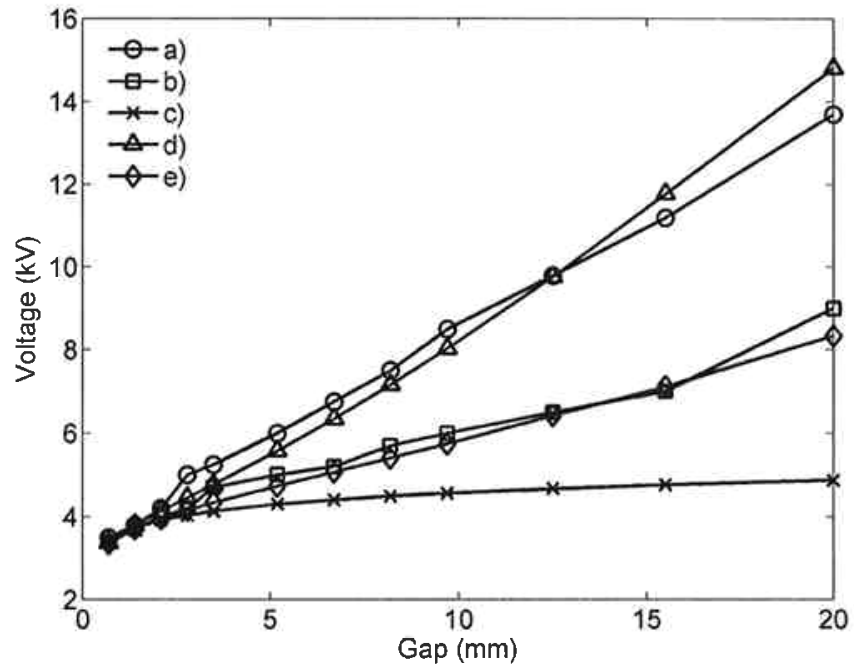

Figure 7. Initiation voltage with an initiation electric field of $9.8 \mathrm{MV} / \mathrm{cm}$. a) measurement using a $20 \mathrm{~mm}$ diameter plane electrode [4], b) measurement using a $45 \mathrm{~mm}$ plane electrode [4], c) theoretical curve from [11], d) computation with $20 \mathrm{~mm}$ plate, and e) computation with $45 \mathrm{~mm}$.

Using the field strength data, the critical volume was calculated for a range of electric field strengths as shown in Figure 9. The critical volume increases if the electric field defining it is between $10 \%$ and $30 \%$ of $\mathrm{E}_{\max }$, and decreases steeply if the field is greater than $40 \%$ of $\mathrm{E}_{\max }$. From Figure 9, the constant critical volume is between $0.4 \mu \mathrm{m}^{3}$ and $2.0 \mu \mathrm{m}^{3}$, a value consistent with Case 1 shown in Figure 6.

From the results of Case 1 and Case 2, the electric fields related to distances and critical volume, rather than maximum field strength, are important. Case 1 and Case 2 are consistent with each other if the initiation occurs within a critical volume between 0.4 and $1.0 \mu \mathrm{m}^{3}$.

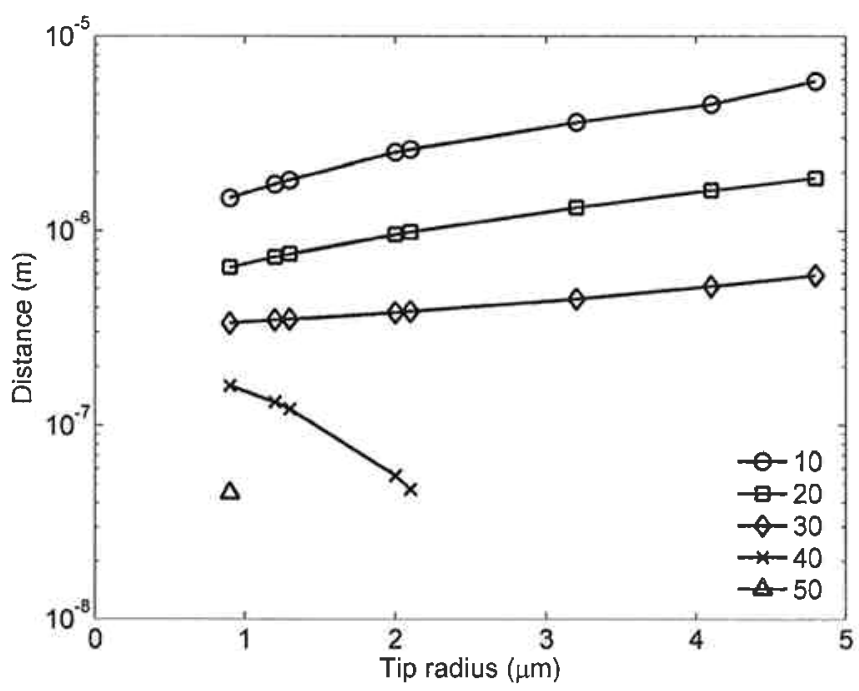

Figure 8. The calculated electric field strength as a function of tip radius for various percentages of $E_{\max }$ with the geometry of Figure 1b). 


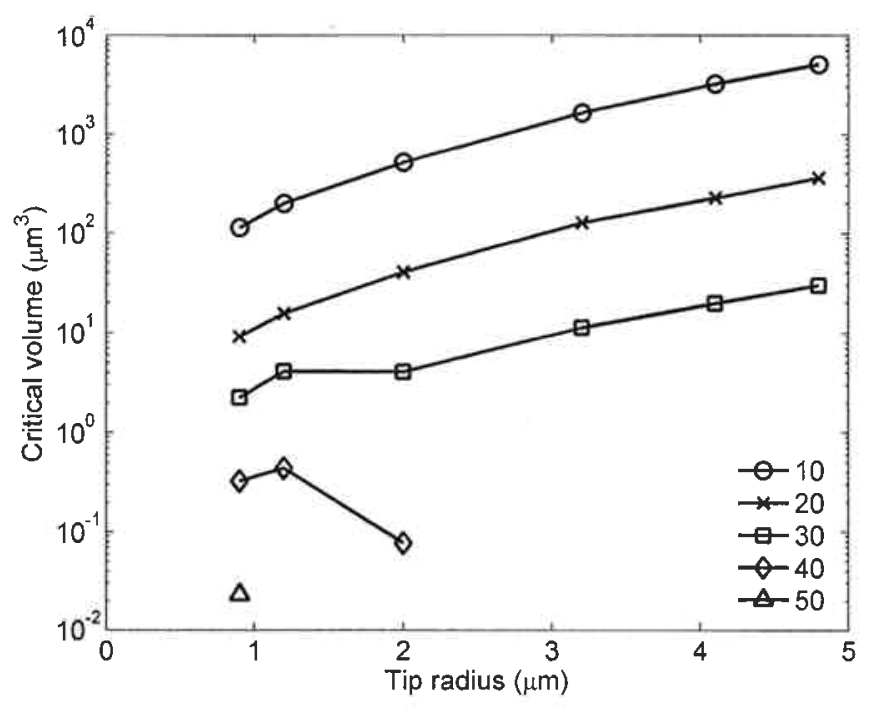

Figure 9. Critical volume with chosen electric fields in Case 2.

\subsection{Electronic Implications}

This analysis shows that the assumption of a critical volume for streamer initiation is consistent with experimental observations of streamer initiation. It addition, it adds detail to the observation by Yamashita, et al. [3] that the calculated region of constant field strength must have some significance in the breakdown process. A key part of the assumption in the critical volume model is that an electron created in the volume has sufficient free path in that volume to gain enough kinetic energy from the electric field that it can stimulate the formation of an avalanche.

At the lowest level of consideration, there appears to be sufficient energy available if it is assumed that the boundary of the critical volume is more than $0.1 \mu \mathrm{m}$ from the electrode tip and the minimum field in the volume is about $7 \mathrm{MV} / \mathrm{cm}$. So, an electron could gain energy of more than $70 \mathrm{eV}$ by traversing the critical volume. As the ionization energy of cyclohexane is between 8.4 and $8.8 \mathrm{eV}[14,15]$, there would be adequate energy available for ionization within that volume.

Given that the critical volume is filled with a liquid, however, it is not just the size of the critical volume, but also the free path of the electron that determines the energy an electron can extract from the applied field before a molecular collision. Therefore, streamer initiation is a more complex process of an energy gain from the electric field and energy losses during collisions with the liquid molecules. These energy loss processes have been studied extensively for radiation detection [16]. While there is no complete set of comparison data for cyclohexane, the liquid used in this work, available information shows that over the range of energies of interest, the free path, and consequently the thermalization distance for an electron, is a function of energy.

For thermal electrons, i.e., those with energy of about 0.025 $\mathrm{eV}$, the free path is $0.03 \mathrm{~nm}$ [15]. For higher energy electrons such as those produced by $\mathrm{x}$-rays with no applied field, the thermalization distance at room temperature is about $6 \mathrm{~nm}$ [17]. Moreover, in hydrocarbons, the majority of the thermalization distance occurs when the electron has energy of a few tenths of an $\mathrm{eV}$ [6]. As the energy of the electron is increased significantly, the effective mean free path increases significantly. Specifically, the effective mean free path for momentum transfer, i.e., for electrons with energy above a few tenths of an $\mathrm{eV}$ but less than the ionization energy, is 1.5-2.6 $\mathrm{nm}$ [18].

The most probable energy loss in solid cyclohexane is in the range 19-24 eV, and the mean free path for $25 \mathrm{eV}$ electron is about $14 \mathrm{~nm}$. The values for the solid phases of hydrocarbons are acceptable approximations for the liquid phase [19].

Because of the complex processes affecting the free path, and because of the energy dependence, it is impossible to assign a specific number to a free path that is experienced by a discharge-initiating electron. Figure 10, however, shows the distance the free path would need to be for the ionization energy of $8.4 \mathrm{eV}$ if the discharge were to initiate near the low field boundary of the critical volume for the data in $[3,4]$. This shows that the free path for ionization would need to be in the single digit nanometer range, which appears to be consistent with the published data on free paths, although no data exists for exactly the situation observed here. With that caveat, this analysis appears to be consistent with the following explanation of the data:

1. If an electron is created in what will be the critical volume during or immediately prior to the initiation of the voltage pulse, the electron can achieve sufficient energy for momentum transfer. This produces the $1^{\text {st }}$ anode mode consistent with published data $[3,4]$.

2. If the electron is produced in the critical volume after the voltage is established, the $2^{\text {nd }}$ anode mode is produced as there is sufficient energy gained by the electron for an electron avalanche to occur.

Finally, it is likely that the maximum free path, rather than the mean free path, is responsible for breakdown initiation. If this is true, the occurrence of the maximum free path links this work with the fluctuation model [20] and the percolation approach [21].
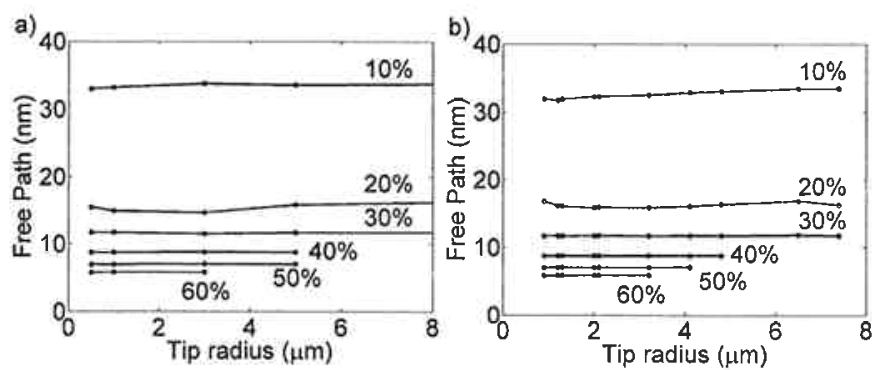

Figure 10. The free path required for an electron to achieve ionization energy in cyclohexane as a function of the tip radius with the percentage of the peak field as a parameter. The calculations in (a) were for the geometry in [3] while those in (b) are for the geometry in [4]. 


\section{CONCLUSION}

The assumption of a critical volume in which an anode discharge is initiated is well established in gaseous breakdown where free paths and interaction cross sections are well known. If one applies the same model to anode initiated electrical breakdown in cyclohexane, one obtains a result that is consistent with the location of the initiation along the point electrode, is consistent with the measured variation of the initiation voltage with electrode radius for the $1^{\text {st }}$ anode mode, is consistent with the constancy of the initiation voltage for the $2^{\text {nd }}$ anode mode, and is consistent with the free path of electrons in cyclohexane for the energies of interest. These results show that this may be a useful model for anode streamer initiation, particularly as the work in fluctuation dynamics and percolation become more refined.

\section{ACKNOWLEDGMENT}

The authors thank the Office of Naval Research for support of this work. In addition, they thank K. Davey for introducing them to software well suited to the solution of this class of problem.

\section{REFERENCES}

[1] A. Beroual, M. Zahn, A. Badent, K. Kist, A. J. Schwabe, H. Yamashita, K. Yamazawa, M. Danikas, W. G. Chadband, and Y. Torshin "Propagation and structure of streamers in liquid dielectrics," IEEE Electr. Insul. Mag., Vol. 14, No. 2, pp. 6-17, 1998.

[2] E. O. Forster, H. Yamashita, C. Mazzetti, M. Pompili, L. Caroli, and S. Patrissi, "The effect of the electrode gap on breakdown in liquid dielectrics," IEEE Trans. Dielect. Elect. Insulation, Vol. 1, No. 3, pp. 440-446, June 1994

[3] H. Yamashita, K. Yamazawa, and Y. S. Wang, "The effect of tip curvature on the prebreakdown streamer structure in cyclohexane," IEEE Trans. Dielect. Elect. Insulation, Vol. 5, No. 3, pp. 396-401, June 1998.

[4] P. Gournay and O. Lesaint, "A study of the inception of positive streamers in cyclohexane and pentane," J. Phys. D: Appl. Phys., Vol. 26, pp. 1966-1974, 1993.

[5] R. E. Hebner, "Measurements of electrical breakdown in liquids," in The Liquid State and its Electrical Properties, Plenum Press, New York, pp. 519-537, 1988.

[6] G. R. Freeman, "Ionization and charge separation in irradiated materials," in Kinetics of Nonhomogeneous Processes - A Practical Introduction for Chemists, Biologists, Physicists, and Material Scientists, J. Wiley \& Sons, G. R. Freeman, ed., New York, pp. 19-25, 1987.

[7] S. K. Venkatesh and M. S. Naidu, "Experimental and theoretical investigations on the statistics of time lags to corona inception and breakdown of SF6 in non-uniform electric fields," in Gaseous Dielectrics, Vol. VIII, Christophorous and Olthoff, Eds. New York: Plenum, pp. 115-160, 1998.

[8] A. L. Kupershtokh and D. I. Karpov, "Stochastic features of liquid dielectric breakdown at small area of positive electrode," IEEE $13^{\text {th }}$ Int. Conf. on Diel. Liquids, pp. 203-206, 1999.

[9] A. P. Ershov and A. L. Kupershtokh, "Fluctuation model of liquid dielectrics breakdown with incomplete charge relaxation," IEEE $11^{\text {th }}$ Int. Conf. on Diel. Liquids, pp. 194-198, 1993.

[10] K. Yamazawa and H. Yamashita, "Calculation of the electric field distribution under the point-plane gap configurations using the FEM," IEEE Conf. on Elec. Insul. and Diel. Phenom., pp. 648-651, 1997.
[11] E. F. Kelley and R. E. Hebner, "The electric field distribution associated with prebreakdown phenomena in nitrobenzene," J. Appl. Phys., Vol. 52, pp. 191-195, 1981.

[12] H. A. Fowler, J. E. Devaney, and J. G. Hagedorn, "Shaping of filamentary streamers by the ambient field," IEEE Conf. on Elect. Insul. and Diel. Phenom., pp. 132-136, 1999.

[13] R. Coelho and J. Debeau, "Properties of the tip-plane configuration," J. Phys. D: Appl. Phys., Vol. 4, pp. 1266-1280, 1971.

[14] W. F. Schmidt, "Electrons in non-polar dielectric liquids," IEEE Trans. Elec. Insu1., Vol. 28, pp. 560-567, 1991

[15] J. P. Guelfucci, et al., "VUV photo-ionization parameters and effective mean free path of epithermal photoelectrons on pure non-polar dielectric liquids" IEEE Trans. Diel. Elec. Insul., Vol. 9, pp. 263-273, 2002.

[16] H. G. Paretzke, "Radiation track structure theory," in Kinetics of Nonhomogeneous Processes - A Practical Introduction for Chemists, Biologists, Physicists, and Material Scientists, J. Wiley \& Sons, G. R. Freeman, ed., New York, pp. 89-170, 1987.

[17] N. Gee and G. R. Freeman, "Free ion yields, thermalization distances, and ion mobilities in liquid cyclic hydrocarbons: Cyclohexane and trans- and cis-decalin," J. Chem. Phys., Vol. 96, pp. 586-592, 1992.

[18] J. P. Guelfucci, et al., "Photo-ionization spectrum, spatial distribution function of photo-electrons and evolution of photo-ionization parameters as a function of excess energy for some non-polar dielectric liquids (Cyclohexane, 2-2 Dimethylbutane, Tetramethysilane and Polymethylsiloxane Oils) irradiated by VUV photons," IEEE $13^{\text {th }}$ Int. Conf. on Diel. Liquids, pp. 104-107, 1999.

[19] J. A. LaVerne and S. M. Pimblott, "Electron energy loss distributions in solid and gaseous hydrocarbon," J. Phys. Chem., Vol. 99, pp. 1054010548,1995

[20] A. L. Kupershtock, "Propagation of streamer top between electrodes for fluctuation model of liquid dielectrics breakdown," IEEE $12^{\text {th }}$ Int. Conf. on Diel. Liquids, pp. 210-213, 1996.

[21] K. Kist, Y. Julliard, R. Badent, and A. J. Schwab, "Streamers and percolation - a new theoretical approach," IEEE Conf. on Elect. Insul. and Diel. Phenom., pp. 474-477, 2000.

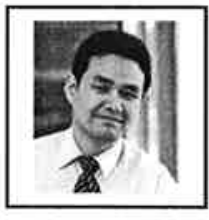

Minkyu Kim was born in Seoul, Korea in 1971. He received his B.S. degree from Chung-Ang University, Seoul, Korea, in 1994 and his M.S. degree from Seoul National University, Seoul, Korea, in 1997, both in electrical engineering. He is currently pursuing his Ph.D degree in electrical enginecring at the University of Texas, Austin, TX.

Previously, he was with Samsung Electronics from 1997 to 2002, and worked in the areas of processing plasmas, plasma physics, and control systems. His research interests include streamer analysis, processing plasmas, plasma physics, and electromagnetic waves from plasmas.

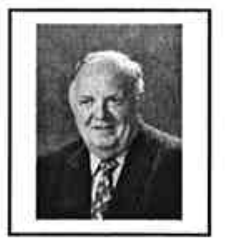

Robert E. Hebner, Ph.D., is Director of the Center for Electromechanics at the University of Texas at Austin. The Center develops technology, primarily novel motors, generators, and suspension components, and teams with companies to get the technology into the market.

Previously, Dr. Hebner was the acting Director of the U.S. National Institute of Standards and Technology (NIST). In addition, he has directed NIST's Electronic and Electrical Engineering Laboratory, a laboratory with a staff of more than 250 . He also worked at the Defense Advanced Research Projects Agency where he developed programs to improve semiconductor manufacturing.

Throughout his career, Dr. Hebner has been active in having authored or coauthored more than one hundred technical papers and reports. He has extensive experience in international technology programs. This work included the modernization of the measurement systems needed to support global trade and the assessment of the effectiveness of government technology programs in stimulating domestic economies. 